\title{
Agreeing on the minimum: An 11-year review of Prescribed Minimum Benefits appeals
}

\author{
P Mngadi, ${ }^{1,2}$ BSc, BTech, MTech, MPH; J Wolvaardt, ${ }^{1}$ BCur, MPH, PhD; E Thsehla, ${ }^{3}$ BSc, MPH \\ ${ }^{1}$ School of Health Systems and Public Health, Faculty of Health Sciences, University of Pretoria, South Africa \\ ${ }^{2}$ Academy of Science of South Africa, Pretoria, South Africa \\ ${ }^{3}$ Council for Medical Schemes, Pretoria, South Africa
}

Corresponding author: J Wolvaardt (liz.wolvaardt@up.ac.za)

\begin{abstract}
Background. Prescribed Minimum Benefits (PMBs) in South Africa (SA) are a set of minimum health services that all members of medical aid schemes have access to regardless of their benefit options or depleted funds. Medical aid schemes are liable to pay for these services. However, $\sim 40 \%$ of all complaints received by the Council for Medical Schemes (CMS) are in relation to PMBs. Individuals/stakeholders who are unsatisfied with judgments on their complaints are allowed to appeal.

Objectives. To determine and describe the pattern of PMB appeals from 1 January 2006 to 31 December 2016.

Methods. This was a descriptive cross-sectional study that utilised the CMS Judgments on Appeals database. Data for PMBs, levels of appeal, judgments, appellants, respondents and medical scheme types were extracted. The CMS's lists of chronic conditions, PMBs and registered schemes were used to confirm PMBs and to categorise schemes as either open (i.e. to all South Africans) or restricted (i.e. only open to members of specific organisations). Data were extracted and frequencies were calculated using Stata software, version 14.

Results. All eligible appeal reports ( $N=340)$ were retrieved and 123 PMB appeals were included in the study (36.2\%). The median number of PMB appeals per year was 11 (interquartile range 9 - 27). Open schemes accounted for $82.1 \%$ of all the PMB appeals. Half of the total appeals $(50.4 \%, 62 / 123)$ were by medical aid schemes appealing their liability to pay for PMBs, and of these $69.4 \%(43 / 62)$ were found in favour of members. The remaining half $(49.6 \%, 61 / 123)$ were appeals by members appealing that schemes were liable to pay, and of these $80.3 \%(49 / 61)$ were found in favour of the medical aid schemes. Treatment options that were scheme exclusions constituted $34.4 \%$ (21/61) of reasons why schemes were found not liable to pay. Various types of cancers and emergency conditions constituted one-quarter of all PMB appeals.

Conclusions. While the pattern is unclear and the extent of the problem is masked, this study shows that a quarter of the conflict resulting in PMB appeals was due to various types of cancers and emergency conditions. Medical schemes should revise their guidelines, policies and criteria for payment of these two services and improve their communication with healthcare providers and members.
\end{abstract}

S Afr Med J 2019;109(7):498-502. DOI:10.7196/SAMJ.2019.v109i7.13683

The private healthcare sector in South Africa (SA) forms part of the country's two-tiered healthcare model (private and public). The private healthcare sector serves $\sim 20 \%$ of the population and consumes $\sim 50 \%$ of total healthcare expenditure. In comparison, the public healthcare sector serves $\sim 80 \%$ of the population and utilises $\sim 48 \%$ of total healthcare expenditure. ${ }^{[1]}$ The majority of the spending $(81 \%)$ in private healthcare is financed through the 82 registered medical aid schemes' prepaid plans and $14 \%$ is paid out of pocket. ${ }^{[1]}$ Medical aid schemes are categorised into open $(n=22)$ and restricted $(n=60)$ schemes. ${ }^{[2]}$ Open medical aid schemes are open to all South Africans, while restricted schemes are restricted to groups such as employees of an organisation. ${ }^{[3]}$ In 2016/17, the 22 open medical aid schemes had 4953180 beneficiaries (this included both principal members and their dependants), while the 60 restricted medical aid

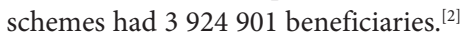

The key role of medical aid schemes in SA, under the Medical Schemes Act (MSA) (Act No. 131 of 1998), ${ }^{[4]}$ is to pay the healthcare expenses of their beneficiaries. This role is based on the agreement that medical aid scheme members pay monthly contributions towards specific scheme benefit plans, which then guarantees payment of their acquired healthcare expenses. ${ }^{[3]}$ Prescribed Minimum Benefits (PMBs) in SA are a set of benefits the purpose of which is to ensure that all members of medical aid schemes have access to certain minimum health services, regardless of their benefit options. ${ }^{[5]}$ PMBs were introduced under the MSA as a policy tool to define the minimum acceptable levels of benefits that medical aid schemes are required to cover. ${ }^{[6]}$ The first goal of defining PMBs was to prevent instances where beneficiaries were deprived of their medical aid scheme cover when sick, and the subsequent use of public sector facilities. The second goal was to enhance efficiency in the allocation of resources between the private and public sectors. ${ }^{[6]}$

All 82 medical aid schemes are legally obliged to pay costs related to the specified diagnosis, treatment and care of PMBs. ${ }^{[5]}$ PMBs are classified into three groups: (i) any emergency medical condition; (ii) a limited set of 270 medical conditions; and (iii) 25 chronic conditions. ${ }^{[5]}$ The role of the Council for Medical Schemes (CMS) is to ensure that medical aid schemes abide by their legal obligations according to the MSA. ${ }^{[4]}$ Even though medical aid schemes are liable to pay for all three groups of PMBs, members and healthcare providers are required to abide by the schemes' protocols and guidelines. These guidelines include the schemes' diagnosis, treatment and care protocols for PMBs and using schemes' designated service providers (DSPs) ${ }^{[7]}$ DSPs are healthcare providers (medical doctors, hospitals, etc.) that medical schemes prefer their members to use when they need diagnosis, treatment or care for PMBs. ${ }^{[7]}$ The contracts with DSPs include agreed-upon standard fees for PMBs. When a member breaches these guidelines, medical aid schemes are exonerated from paying. Appeals for PMB conditions therefore arise when schemes refuse to pay in full or insist on co-payments for diagnosis, treatment, management and care of PMBs. Some of the reasons 
linked to this refusal of payment include: (i) members voluntarily using non-DSPs for health services; (ii) members obtaining treatment that forms part of the scheme's exclusion or is not part of the treatment protocol for a specific PMB; (iii) members opting for treatment that has co-payment requirements; and (iv) members being in arrears in terms of their monthly membership contributions. $^{[5]}$ Both the medical aid schemes and the PMBs are regulated under the MSA. ${ }^{[4,6]}$ The MSA designates the CMS as the responsible body to protect the interests of members of medical aid schemes. ${ }^{[8]}$ Members of medical aid schemes therefore have a right to lodge complaints with the CMS in relation to PMBs if payments have been wrongly denied. The medical aid schemes' complaint and appeal process in SA is divided into five levels: $(i)$ the internal medical aid scheme dispute committee; (ii) the CMS Registrar; (iii) the CMS Appeal Committee; (iv) the CMS Appeal Board (at a cost of ZAR2 800 by the appellant); and $(v)$ the High Court. ${ }^{[8]}$

All stakeholders (including medical aid schemes, members and healthcare providers) have a right to appeal the rulings and judgments. In 2009, the CMS noted with concern that there had been a dramatic increase from $9.3 \%$ to $25.4 \%$ in the number of complaints in relation to PMBs. ${ }^{[9]}$ In an effort to identify issues leading to this increase, the CMS undertook an evaluation, the findings of which revealed high non-compliance by medical aid schemes in terms of payment for PMBs. Medical aid schemes also confirmed their noncompliance. ${ }^{[9]}$ As part of the intervention a task team was formed, and it developed a code of conduct for ensuring full compliance with the provisions of the PMBs. A decrease in PMB complaints was then seen during the period 2009/10. ${ }^{\left[{ }^{[9]}\right.}$ However, despite this intervention, complaints in respect of PMBs increased to $40 \%$ by 2011/12. ${ }^{[10]}$ While the CMS publishes summaries of the PMB complaints patterns in its annual reports, there are no published data on the patterns of PMB appeals in SA. ${ }^{[2]}$

Understanding the pattern of PMB appeals in the SA private health sector could benefit both members and medical aid schemes and could contribute to the reduction of wrongful denials of payments regarding PMBs.

\section{Objectives}

To determine and describe the pattern of PMB appeals in SA from 1 January 2006 to 31 December 2016.

\section{Methods}

Study design and data collection

This was a descriptive cross-sectional study. Secondary data were obtained from the CMS
Judgments on Appeals database between 2006 and 2016. All reports were retrieved.

The following data were extracted: (i) the basis of the appeal; (ii) whether the condition was a PMB; (iii) the level of the appeal process (CMS Appeal Committee or Appeal Board or High Court); (iv) the final judgment (upheld or overturned or no judgment); $(v)$ appellants and respondents; (vi) the type of medical aid scheme (open or restricted); and (vii) the year in which the judgment was made. The CMS's lists of PMBs and the 25 chronic medical conditions were consulted to confirm whether conditions were PMBs. Also, the CMS's list of registered medical aid schemes was used to categorise medical schemes as either open or restricted.

\section{Data analysis}

The collected data were entered onto an Excel spreadsheet (2016, version 16.0.4229.1002;
Microsoft, USA) and coded. Descriptive statistics (percentages and frequencies) were used to summarise categorical data, while medians and interquartile ranges (IQRs) were used for numerical data. Stata version 14 software (StataCorp, USA) was used for analysis.

\section{Ethical considerations}

Permission to utilise the data was received from the CMS's Chief Executive Officer and ethics approval was granted by the University of Pretoria Faculty of Health Sciences Research Ethics Committee (ref. no. 551/2017).

\section{Results}

Of the 340 eligible appeals on the CMS database, a total of 123 (36.2\%) were PMB appeals and therefore included in the study. Appeals that did not meet the inclusion criteria were excluded (Fig. 1).

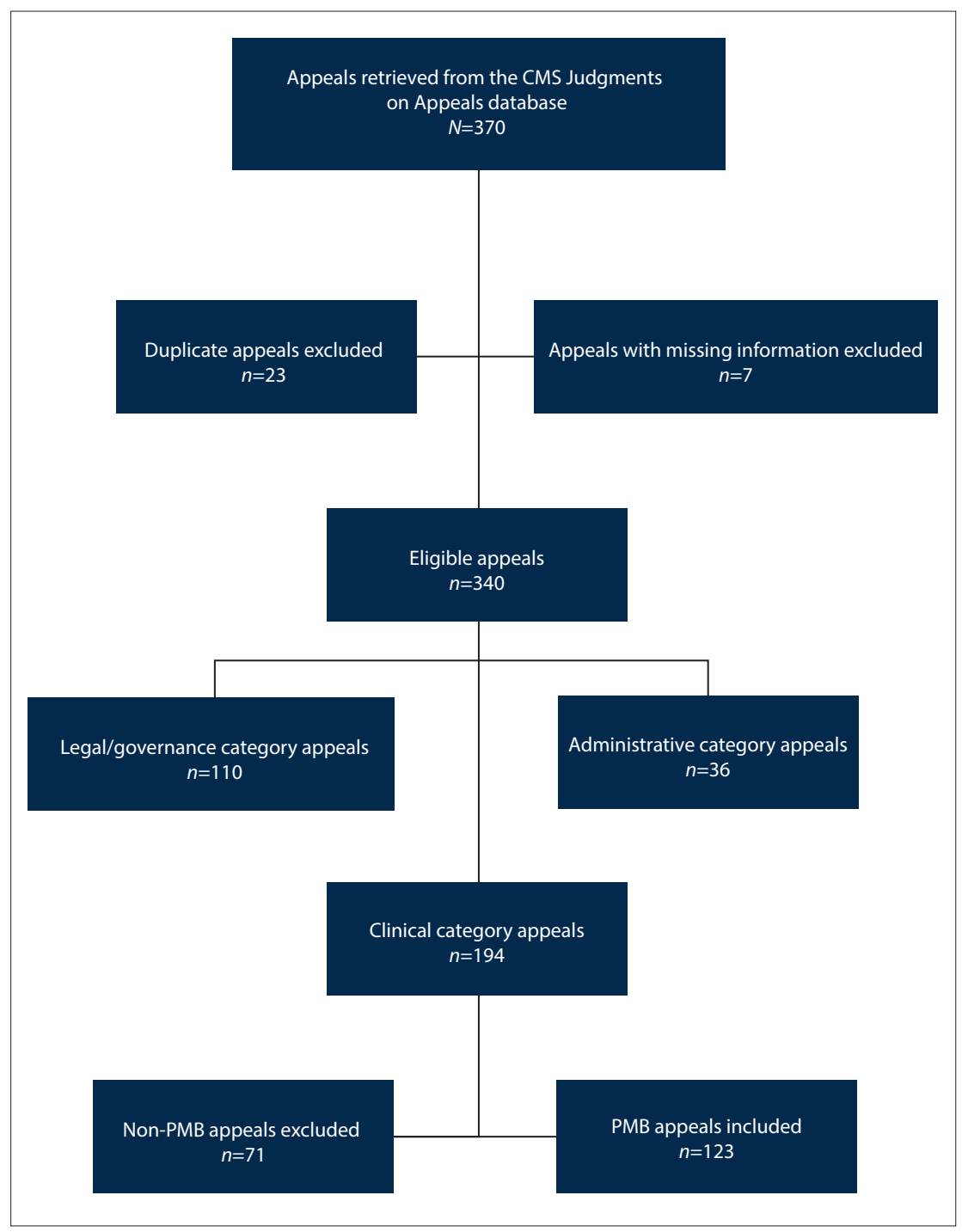

Fig. 1. Selection process of PMB appeals for 1 January $2006-31$ December 2016. (PMB = Prescribed Minimum Benefits; CMS = Council for Medical Schemes.) 
The median number of PMB appeals per year was 11 (IQR 9 - 27). The number of $\mathrm{PMB}$ appeals throughout the 11-year period of analysis is shown in Table 1.

Of the 123 appeals, $50.4 \%$ (62/123) were by medical aid schemes appealing their liability to pay for the PMBs, and of these $69.4 \%$ (43/62) were found in favour of members and 30.6\% (19/62) in favour of medical aid schemes. The remainder of the PMB appeals (49.6\%, 61/123) were by members (and one healthcare provider) appealing that medical aid schemes were liable to pay for PMBs. Of these appeals, $80.3 \%$ (49/61) were found in favour of the medical aid schemes. Only $11.5 \%$ (7/61) were overturned to favour members, and 'no judgments' were made for $8.2 \%$ (5/61) of these appeals. The five main reasons that medical aid schemes cited for refusing payment were: (i) treatments used by members were scheme exclusions or not part of the treatment protocol for a PMB condition (41.0\%); (ii) members used non-DSPs to obtain treatment and care for PMBs (14.8\%); (iii) treatment chosen by members required co-payments (6.7\%); (iv) depleted benefit funds (5.0\%); and ( $v$ ) off-label use of treatment (i.e. not complying with the instructions for administering the drugs/medication) by healthcare providers in treating members $(3.3 \%)$.

A range of $\mathrm{PMB}$ conditions were identified, and the top 10 most commonly appealed conditions are listed in Table 2. The top three conditions included in the appeals were cancer (18.0\%), emergency conditions ( $8.1 \%$ ) and pregnancy $(6.5 \%)$. Four conditions constituted the fewest appeals in this list: heart conditions, fractures, hyperlipidaemia and respiratory conditions (all 3.3\%).

More than half $(60.0 \%, 6 / 10)$ of the 10 emergency conditions appealed were disputes about whether they were genuine emergencies for members to have involuntarily acquired non-DSPs' services. Two of these emergencies were appeals where healthcare providers had claimed a special fee (termed the Modifier 0019 fee) for premature baby emergencies. Half of the judgments on these appeals found in favour of the members and half were in favour of medical aid schemes.

There were three types of appellants and four types of respondents identified in the PMB appeals over the period of analysis. Healthcare providers played a minor role with regard to being either an appellant or a respondent (Table 3 ).

Overall, in terms of type of schemes the appeals were linked to, open medical aid schemes accounted for $82.1 \%(101 / 123)$ of the PMB appeals, while restricted medical aid schemes accounted for $17.9 \%$ (22/123). The majority $(92.7 \%, 114 / 123)$ of PMB appeals did not progress beyond the first level of appeal (the Appeal Committee). No PMB appeals were recorded as progressing to the High Court.

\section{Discussion}

This study is the first to review appeals on PMBs in SA beyond a single CMS reporting cycle. Over the period of analysis, no clear pattern was found in terms of the numbers of appeals increasing or decreasing. The number of PMB appeals was lowest in 2009, and this coincided with the year that the Registrar of the CMS implemented a code of conduct for PMB regulation compliance by medical aid schemes. ${ }^{[9]}$ This code of conduct was a result of a notable increase in the number of $\mathrm{PMB}$ complaints and confirmed non-compliance with PMB regulation by medical aid schemes prior to this date. ${ }^{[9]}$ Despite implementation of this intervention, after 2009 the number of PMB appeals fluctuated over the period of analysis, with 2012 recording the highest number to date. The year 2013 had the second-lowest number of PMB appeals, and this corresponded with the Registrar's second intervention. ${ }^{[11]}$ The complaints lodging system was revised and healthcare providers were instructed to first exhaust all internal
Table 1. Annual numbers of published PMB appeals $(N=123)$

\begin{tabular}{ll}
\hline Year of judgment & Frequency, $\boldsymbol{n}$ (\%) \\
\hline 2006 & $3(2.4)$ \\
2007 & $3(2.4)$ \\
2008 & $8(6.5)$ \\
2009 & $2(1.6)$ \\
2010 & $15(12.0)$ \\
2011 & $16(13.0)$ \\
2012 & $28(23.0)$ \\
2013 & $3(2.4)$ \\
2014 & $7(6.0)$ \\
2015 & $15(12.0)$ \\
2016 & $23(18.7)$ \\
PMB = Prescribed Minimum Benefits. & \\
&
\end{tabular}

Table 2. Top $10 \mathrm{PMB}$ conditions appealed $(N=123)$

\begin{tabular}{ll}
\hline Condition & Frequency, $\boldsymbol{n}$ (\%) \\
\hline Cancer & $22(18.0)$ \\
Emergency conditions & $10(8.1)$ \\
Pregnancy & $8(6.5)$ \\
Crohn's disease & $6(5.0)$ \\
Leukaemia & $6(5.0)$ \\
Hypertension & $5(4.1)$ \\
Heart conditions & $4(3.3)$ \\
Fractures & $4(3.3)$ \\
Hyperlipidaemia & $4(3.3)$ \\
Respiratory difficulties & $4(3.3)$ \\
PMB = Prescribed Minimum Benefits. &
\end{tabular}

Table 3. Appellants and respondents on PMB appeals $(N=123)$

\begin{tabular}{ll}
\hline & Frequency, $\boldsymbol{n}(\%)$ \\
\hline Appellants & \\
Medical aid schemes & $63(51.0)$ \\
Members & $59(48.0)$ \\
Healthcare providers & $1(1.0)$ \\
Respondents & \\
Medical aid schemes & $58(47.2)$ \\
Members & $57(46.3)$ \\
Healthcare providers & $4(3.3)$ \\
CMS & $4(3.3)$ \\
PMB = Prescribed Minimum Benefits; CMS = Council for Medical Schemes.
\end{tabular}

medical aid scheme resolution processes before approaching the CMS. ${ }^{[11]}$ This intervention may explain the reduced number of complaints referred to the CMS in 2013 and 2014. ${ }^{[11]}$

However, by 2015 and 2016 there was a rise in PMB appeals referred to the CMS. Cancers of various types and emergency conditions constituted a large proportion of PMB conditions that members appealed and medical aid schemes contested paying. This finding differs from what was reported in the USA, where mental health conditions were the most rejected claims for payment by health insurers. ${ }^{[12]}$ Both of these PMB categories (cancers and emergency conditions) are potentially life-threatening if not treated promptly, and cancers may require long-term and costly treatment. In terms of emergency conditions, the criteria used by medical aid schemes in deciding whether a condition was a real emergency seemed to be the main area of contention. The schemes' varying interpretations of and 
conclusions regarding an emergency condition $\mathrm{v}$. an urgent medical condition are a challenge for members. The findings from this study appear to support the US study, which showed that in the denial of payments, schemes' interpretations of their guidelines sometimes vary. ${ }^{[12]}$ The CMS PMB list defines an emergency condition as a sudden health condition in need of immediate treatment, but schemes appear to interpret this definition in a manner that favours avoidance of their paying. ${ }^{[5]}$

Half of the judgments on emergency conditions favoured members and half favoured schemes, further highlighting this contention and confusion. Unfortunately for members, when in their perspective a medical condition becomes an emergency they are likely to visit the nearest healthcare provider without confirming whether this is a DSP. The main priority for members in an emergency is to access medical care as soon as possible, before further and/or more permanent damage is sustained. This reasoning supports the CMS's justification for why emergencies need prompt treatment. ${ }^{[5]}$ In addition, the insurance afforded during emergencies is likely to be one of the reasons why people join medical aid schemes. Payment by medical aid schemes in such cases is therefore crucial to avoid out-of-pocket payment by members.

In the instances where members appealed, medical aid schemes were confirmed as liable to pay for the members' PMB-related costs in the majority of the PMB appeals. The judgments on these appeals were not surprising, because refusals to pay for legitimate $\mathrm{PMB}$ claims strengthen the perception that schemes use claim denials as a way to save money. Of concern, however, is that it is not known how many legitimate PMB claims are rejected without members appealing. These study findings and concerns are corroborated by two studies in the USA which showed that medical aid schemes reject members' legitimate medical claims purely to save money, and that most members do not appeal rejections of their medical claims. ${ }^{[1,13]}$ One of these two studies found that only $7 \%$ of members appealed beyond the first level of appeal, while in the second study, $4 \%$ of the complaints were appealed higher than the first level. ${ }^{[12,13]}$

Medical aid schemes were largely successful in their own appeals. Overall, members were less successful, as the majority of the claims were judged in favour of the medical aid schemes.

While medical aid schemes are liable by law to pay for PMBs, the responsibility also lies with members and healthcare providers to follow the schemes' rules and guidelines in terms of diagnosis, treatment and care for PMBs. ${ }^{[5]} \mathrm{A}$ large proportion of members who appealed were found to be at fault. Most commonly the members had used non-DSPs to provide healthcare, or their healthcare providers administered PMB treatments that schemes were not liable to pay for, so that members were liable for the co-payment. In these instances, medical aid schemes are within the law to refuse to pay in full or to pay only a portion of a claim. As a result, members face a financial burden, as they have to make out-of-pocket payments. The use of non-DSPs exacerbates the problem, as these healthcare providers may charge substantially more than the schemes' DSP rates. ${ }^{[7]}$ Such noncompliance could be due to medical aid schemes not communicating these processes properly; alternatively, members and healthcare providers may not be familiarising themselves with the processes.

The Appeal Committee of the CMS reviewed the largest proportion of PMB appeals, with very few reviewed by the CMS Appeal Board and none reaching High Court level. The escalation process of appeals in SA is similar to that in the USA, where an appeal can potentially be appealed from level one all the way up to level five, with level five being the Court for both countries. ${ }^{[12,13]}$ The Appeal Committee, which is at level three of the SA appeal process, corroborates the US study findings that members escalate their appeals up to level two and three because judgments are normally not in their favour at level one. ${ }^{[12]}$ The finding that only a few of the appeals were at Appeal Board level was not unexpected, because of the cost associated with referring cases to the Appeal Board. ${ }^{[8]}$ Furthermore, as payment is the main area of contention in PMB appeals, additional costs are likely to prevent members from appealing further than the Appeal Committee. The additional costs and time needed for the higher levels of the appeal process (Appeal Board and High Court) are likely to affect members more than medical aid schemes or healthcare providers. The negative implication of the appeal-related costs is that even if members are unsatisfied with an Appeal Committee judgment, they may have no choice but to accept it.

No obvious difference was found between the medical aid schemes and members in their roles as appellants. Members are justified in appealing PMB payments in view of the rising out-of-pocket payments that have resulted from SA's unsustainable healthcare financing system and that contribute to the financial burden of households. ${ }^{[14]}$ Furthermore, as PMBs include chronic conditions, denial of payment could have an effect on life-threatening illnesses, which in turn is a motivation for members to appeal. ${ }^{[5]}$ The right to appeal does not necessarily result in an actual appeal. ${ }^{[9]}$ In SA it is difficult to ascertain how many members could potentially have appealed but did not do so over the 11-year period analysed. This lack of information is a challenge, because it is possible that there were potentially more legitimate $\mathrm{PMB}$ appeals that members ultimately paid out of pocket instead of appealing.

Few of the PMB appeals were linked to restricted medical aid schemes, despite the predominance of these schemes. ${ }^{[2]}$ One explanation could be the number of beneficiaries in open schemes ( $N=4953180)$ as opposed to restricted schemes ( $N=3924901)^{[2]}$ Owing to their small number of beneficiaries per scheme (average of 65415 beneficiaries per scheme), it is possible that the 60 restricted medical aid schemes communicate more effectively with their members. In comparison, the 22 open medical aid schemes average 225144 beneficiaries per scheme. These differences in terms of numbers per scheme could be a factor contributing to the number of PMB appeals. Other possible explanations may be that restricted medical aid schemes have more effective PMB internal dispute resolution processes, or poor knowledge of the 'outside' appeal process on the part of members of the restricted medical aid schemes. This finding highlights a need for future research to understand why restricted medical aid schemes have fewer PMB appeals, taking into consideration the differences in the number of beneficiaries.

\section{Study strengths and limitations}

The strength of the present study is the 11 years' worth of data that were available. The study did not take into consideration the size (i.e. number of beneficiaries) of the two types of medical aid schemes in the analysis. Also, based on the low number of appeals compared with the number of beneficiaries, annual rates were not calculated and only the annual actual numbers were analysed. The study also did not take into consideration appeals that were appealed more than once, as the CMS does not upload repeat appeals to the Judgments on Appeals database.

Finally, the use of secondary data is a limitation because the data were originally collected for a different objective to what they were used for in this analysis.

\section{Conclusions}

The CMS's interventions to reduce the number of appeals have been effective but short-lived. The actual extent of the problem is masked, as it is likely that not all members who have a rejected PMB 
claim appeal the decision. While the overall numbers of appeals are small in relation to the size of medical aid scheme membership, the consequences to individuals are substantial. Research is needed to understand the measures required to prevent conflict with regard to the various types of cancers and emergency conditions that constituted one-quarter of all PMB appeals. Medical schemes should revise their guidelines, policies and criteria for payment of these two services and improve their communication with healthcare providers and members.

Declaration. This study was conducted as part of a Master of Public Health programme (PM).

Acknowledgements. The authors thank the CMS for permission to use their records as data.

Author contributions. PM, JW and ET conceptualised the project, and PM wrote the protocol under the guidance of JW and ET. PM collected and analysed the data, and ET provided guidance during the data analysis phase. PM and JW drafted the first version of the article and ET commented on it. All authors read and approved the final manuscript. Funding. None.

Conflicts of interest. ET is employed by the CMS.
1. RH Bophelo. A glimpse into the South African healthcare industry. https://www.rhbophelo.co.za/ understand-the-south-african-healthcare-industry/ (accessed 29 May 2019).

2. Council for Medical Schemes. Annual report 2016/2017. https://www.medicalschemes.com/files/ Annual\%20Reports/CMSAnnualReport\%2020162F17.pdf (accessed 29 May 2019).

3. Fedhealth. How do medical schemes work? https://www.fedhealth.co.za/medical-aid-questions definition-of-medical-aid-scheme/ (accessed 29 May 2019).

4. South Africa. Medical Schemes Act No. 131 of 1998. https://www.gov.za/sites/default/files/gcis document/201409/a131-98.pdf (accessed 29 May 2019).

5. Council for Medical Schemes. Prescribed Minimum Benefits. https://www.medicalschemes.com/ medical_schemes_pmb/ (accessed 29 May 2019).

Council for Medical Schemes. Prescribed Minimum Benefits review. http://www.medicalschemes. com/files/PMB\%20Review/PMB\%20Review\%20consultative\%20doc.pdf (accessed 29 May 2019).

7. Council for Medical Schemes. Designated service providers. https://www.medicalschemes.com/ medical_schemes_pmb/designated_service_providers.htm (accessed 29 May 2019).

8. Council for Medical Schemes. The Appeals Committee. https://www.medicalschemes.com/Content. aspx?136 (accessed 29 March 2019).

. Council for Medical Schemes. Annual report 2009/2010. https://www.medicalschemes.com/files/ Annual\%20Reports/CMSAR2009_2010.pdf (accessed 29 March 2019).

10. Council for Medical Schemes. Annual report 2011/2012. https://www.medicalschemes.com/files/ Annual\%20Reports/CMSAR20112012.pdf (accessed 29 March 2019).

11. Council for Medical Schemes. Annual report 2013/2014. https://www-medicalschemes.com/files Council for Medical Schemes. Annual report 2013/2014. https://WW
Annual\%20Reports/AR2013_2014LR.pdf (accessed 29 March 2019)

12. Olaniyan O. Reducing lost revenue from inpatient medical-necessity denials: A data-driven approach can help hospitals limit payment denials that are based on questions of whether an admission was medically necessary. Healthc Financ Manage 2015;69(2):74-79.

3. USA Government. Medicare health plan appeals. www.medicare.gov/claims-appeals/file-an-appeal/ appeals-if-you-have-a-medicare-health-plan (accessed 15 June 2018).

14. Jobson M. Structure of the Health System in South Africa. Johannesburg: Khulumani Support Group. https://khulumani.net/active-citizens/item/1164-the-health-system-in-south-africa-actions-tosecure-the-right-to-health-in-south-africa.html (accessed 29 March 2019). 